Volume 40

\title{
The application of genomic technology to combat parasitic disease
}

\author{
Edited by KEITH MATTHEWS, SARA MELVILLE and MIKE TURNER \\ CO-ORDINATING EDITOR L. H. CHAPPELL
}

\section{Preface}

Over the last several years there has been considerable support for the provision of genomic and postgenomic technology in parasitology from the funding agencies. This has ranged from the large-scale funding of complete genome sequencing efforts, and high throughput approaches for assaying mRNA and protein expression profile, to more laboratory-scale efforts examining gene and protein expression in particular parasite life cycle stages, or where important traits are expressed (for example in drug resistant parasite lines).

These approaches all provide fundamentally important information in terms of parasite biology, giving insight into particular infection processes, or more holistic information on the complete operations of the parasitic organism. Importantly however, the value of genomic and post-genomic information is not restricted to the 'model' parasites where there is already a large set of basic biological information. Instead, many of the technologies have the potential to be of immense value where there are less existing data, and give opportunities for comparison between different parasites, or related non-parasitic organisms. This 'triangulation' of information can reveal processes that are conserved, or which differ between parasites and their hosts and hence can highlight possible areas to target in parasite control strategies.

Despite these opportunities, the successful application of genomic, post-genomic, and functional genomic approaches to problems of direct relevance to parasitic disease remains rare. The aim of this symposium was to showcase the use of a diverse range of modern approaches to further parasite prevention and cure. Importantly the meeting did not focus on one group of parasites or only the established model organisms. Rather, the papers presented highlighted the application of different approaches to gain a range of information, including basic parasite biology and further understanding and exploitation of the hostparasite interaction. The presentations covered a wide range of parasites from those of importance in human disease, to those of enormous economic relevance through their impact on animal health.

The symposium was not just a study of parasites and parasite molecules. For example, the keynote presentation by Barbara Burleigh (Harvard University) elegantly illustrated how the availability of genomes and genomic resources from mammalian hosts can be exploited to understand parasitic processes from the host's standpoint (in this case host cell penetration by Trypanosoma cruzi). Another viewpoint of host cell presentation was presented by Jonathan Wastling (Glasgow, UK), who described the protein components that comprise the rhoptery of Toxoplasma gondii, an organelle specialised for host cell invasion. This ability to link parasitic processes and host and vector responses at the level of the whole genome is immensely powerful, but presents huge challenges for the bioinformaticians who must to make sense of the resulting avalanche of data. Matt Berriman (Sanger Institute, UK) provided a glimpse of how these challenges are being met and how the datasets can be analysed and compared by non-specialists to address complex questions. Two alternative approaches for gene function analysis were illustrated by Mark Field (Imperial College, 
UK) and Martin Shirley (Institute of Animal Health, Compton). With an available genome and a powerful toolbox of reverse genetic methodology, Mark Field described the efforts to assign function to the many genes of unknown function in Trypanosoma brucei by systematic ablation using RNA interference combined with standardized phenotypic analysis. Contrasting with this, Martin Shirley demonstrated how the prior availability of parasites with particular phenotypes of importance in Eimeria spp. (e.g. variable traits such as drug resistance and parasite attenuation) can be used to identify genes involved in each process by combining classical genetics and genome sequence analysis. High quality microarray and proteomic analyses of such diverse parasites as schistosomes (Karl Hoffmann, Cambridge, UK), and Dictyocaulus viviparus (Jacqui Matthews and Rob Beynon, Liverpool, UK) revealed the broad applicability of many of the methodologies for the study of parasitic infection processes in relation to the host response and illustrated their successful application to organisms where experimental tractability remains challenging, and a genome dataset is either incomplete or missing. An alternative strategy to the study of nematode parasites of veterinary importance was illustrated by John Gilleard (University of Glasgow) who explained how the wealth of genetic information and tools for the free living nematode Caenorhabditis elegans can be exploited to study the function of parasite molecules, or regulatory sequences outwith their normal environment. Finally, Nick Westwood (St Andrews University, UK) took us away from parasite molecules completely, and beautifully described the immense potential of high throughput small chemical screens for parasite function, using Toxoplasma motility as an assay.

The broad biological range of the meeting, with balanced coverage of both protozoan and helminth parasites, was deliberate. Parasitologists study organisms that cause disease, and the challenge and responsibility for all of us is applying the basic knowledge gained through studying these fascinating organisms to delivering therapies. This is obvious, but can be lost in the excitement of the many new insights into basic biological processes that parasites offer. Interestingly, the diversity of approaches taken in the papers presented in the meeting illustrated the interdisciplinary value of much of the new technology. Genes are no longer studied in isolation; rather the interaction of cohorts of genes and proteins involved in particular processes can be studied as a group. Moreover, high throughput approaches and the capacity for cross comparison between biological datasets from different organisms has liberated the study of parasites previously limited by biological tractability or the availability of source material.

The symposium was organised by the British Society for Parasitology and kindly hosted at The Linnaean Society in London. It received generous support from the Wellcome Trust and Cambridge University Press for which we express our thanks. In total, 9 papers were presented orally on the day, one of which (by Dr Mark Field) was kindly delivered at very short notice. The symposium was extremely well attended by delegates ranging from senior academics to those just starting their graduate studies and was widely agreed to be both enjoyable and informative. By illustrating the breadth of applicability of genomic technology in a diverse range of parasitic organisms, the organisers hoped to avoid a focus on technology for its own sake. Rather, our aim was to illustrate and stimulate the potential application of these approaches to the study of parasitic diseases, their prevention, diagnosis, and therapy. 